TRANSACTIONS OF THE

AMERICAN MATHEMATICAL SOCIETY

Volume 147, February 1970

\title{
ON A SECOND ORDER NONLINEAR OSCILLATION PROBLEM
}

\author{
BY \\ C. V. COFFMAN( $\left.{ }^{1}\right)$ AND J. S. W. WONG
}

1. Introduction. We are here concerned with the oscillatory behavior of solutions of the following second order nonlinear differential equation:

$$
y^{\prime \prime}+y F\left(y^{2}, x\right)=0,
$$

where the function $F(t, x)$ is continuous and nonnegative for $t \in[0, \infty), x \in(0, \infty)$.

It will be tacitly assumed here that every locally defined solution of (1) is continuously extendable throughout the entire nonnegative real axis. This will be the case if for example one requires that for fixed $t, F(t, x)$ satisfies a uniform Lipschitz condition in some neighborhood of every $x \in[0, \infty)$. (See Hastings [3], and Coffman and Ullrich [2].) Actually, this tacit assumption can easily be removed, see the remarks at the end of the paper. A nontrivial solution of (1) is said to be nonoscillatory if for every $a>0$ the number of its zeros in $[a, \infty)$ is finite, and it is said to be oscillatory otherwise. Different from the linear equation, when $F(t, x)$ is independent of $t$, the nonlinear equation may possess solutions of either kind. In view of this, one is led to consider the following types of oscillation and nonoscillation conditions; namely, those which guarantee all solutions of (1) oscillate and its converse, i.e. the existence of one nonoscillatory solution, and those which guarantee all solutions of (1) do not oscillate and its converse, i.e. the existence of one oscillatory solution. The first type of oscillation and nonoscillation conditions have been the centre of considerable amount of research and there are a number of results available for equation (1) or similar equations. For an expository account on this subject, we refer the reader to Wong [12], where other references may be found. An excellent discussion on the nature of oscillatory and nonoscillatory solutions may also be found in the papers by Moore and Nehari [9], and Nehari [10]. The second type of oscillation and nonoscillation conditions have received little attention until recently. The prototype of equation (1) is the following generalized Emden-Fowler equation:

$$
y^{\prime \prime}+p(x) y^{\gamma}=0,
$$

where $\gamma \geqq 1$ is the quotient of two odd integers. For equation (2), Jasny [4] and Kurzweil [7] have established the following result on the existence of one oscillatory solution:

Received by the editors February 3, 1969.

(1) The research of the first-named author was supported by NSF GP-7662.

Copyright (C) 1970, American Mathematical Society 
THEOREM A. Let $\gamma>1$. If $x^{(\gamma+3) / 2} p(x)$ is nondecreasing in $x$, then equation (2) has an oscillatory solution.

Jasny and Kurzweil's result is complemented by the following theorem of Kiguradze [5]:

THEOREM B. If $x^{(\gamma+3) / 2+\varepsilon} p(x)$ is nonincreasing in $x$, for some $\varepsilon>0$ then equation (2) is nonoscillatory, i.e. all solutions are nonoscillatory.

Very recently, Nehari [11] obtained a result which is an improvement of the result of Kiguradze [5], namely,

THEOREM C. If $(x \log x)^{(\gamma+3) / 2} p(x)$ is nonincreasing in $x$ for sufficiently large $x$, then equation (2) is nonoscillatory.

In [11], Nehari also initiated the study of obtaining similar results for the more general equation (1). Part of the difficulty of such an extension lies in the fact that the proofs of Theorems $\mathrm{A}, \mathrm{B}$, and $\mathrm{C}$ depend heavily on the form of the function $F(t, x)$ in that it is separable as a product of functions of $t$ and $x$. Using some ingenious differential identities and inequalities, Nehari [11] obtained the following nonoscillation result for equation (1) corresponding to Theorem B for equation (2).

THEOREM D. Let $G(t, x)$ be defined by

$$
G(t, x)=\int_{0}^{t} F(s, x) d s .
$$

If (i) for fixed $x, F(t, x)$ is a nondecreasing function of $t$ and (ii) for some positive $\varepsilon$ and all positive $\alpha, x G\left(\alpha x^{1+\varepsilon}, x\right)$ is nonincreasing for $x \in[a, \infty)$ for some $a>0$, then equation (1) is nonoscillatory.

Questions were open as to whether (a) a similar result for equation (1) corresponding to Theorem A for equation (2) holds, and (b) Theorem D may be improved to include Theorem $\mathrm{C}$ as a special case. The purpose of the present work is to answer these questions in the affirmative, thus completing the extension of Theorems $\mathrm{A}$ and $\mathrm{C}$ to the more general equation (1). In fact, we prove a little more than that stated above, and we refer the reader to the last section where a discussion of these details will be given.

2. Oscillation theorem. The desired extension of Theorem A to the nonlinear equation (1) is the following result:

THEOREM 1. Let $F(t, x)$ and $G(t, x)$ be given as above. Suppose that (i) there exist constants $x_{0}, M, c>0$ and $K \geqq 1$ such that

$$
x^{2} F(\alpha x, x) \geqq \frac{1}{4}+c, \quad x \geqq x_{0}, \alpha \geqq M,
$$

and

$$
G(t, x) \leqq K t F(t, x), \quad x \geqq x_{0}, t \leqq M x,
$$


and (ii) for every $\alpha>0$ the function $x G(\alpha x, x)$ is nondecreasing in $x$ for $x \geqq x_{0}$. Then equation (1) has an oscillatory solution.

The proof will be carried out in a series of three lemmas each of which may be of interest in itself. In each of these, we assume without explicit mention, that the hypotheses of the above theorem hold.

LEMMA 1. Let $y(x)$ be a nonoscillatory solution of (1). Then

$$
\liminf _{x \rightarrow \infty} x^{2} F\left(y^{2}(x), x\right) \leqq \frac{1}{4}
$$

Proof. Suppose that (6) fails, then there must exist a $\delta>0$ and an $x_{1} \geqq x_{0}$ such that for $x \geqq x_{1}$

$$
x^{2} F\left(y^{2}(x), x\right) \geqq(1+\delta) / 4 .
$$

But then $y(x)$ satisfies a linear equation:

$$
y^{\prime \prime}+p(x) y=0
$$

where $p(x) \geqq(1+\delta) / 4 x^{2}$, for $x \geqq x_{1}$. Since equation (7) is oscillatory when $p(x)$ $=(1+\delta) / 4 x^{2}$, the given solution $y(x)$ must also be oscillatory by the Sturm Comparison Theorem. This contradicts the assumption that $y$ is nonoscillatory. Thus (6) must hold.

LEMMA 2. Let $y(x)$ be a nonoscillatory solution of (1). Then there exists a positive constant $B$, independent of the initial values of $y(x)$ and $y^{\prime}(x)$ such that

$$
\limsup _{x \rightarrow \infty}\left|x^{1 / 2} y^{\prime}(x)-\frac{1}{2} x^{-1 / 2} y(x)\right| \leqq B
$$

Proof. Let $\phi(x)=x^{1 / 2} y^{\prime}(x)-\frac{1}{2} x^{-1 / 2} y(x)$. We note first that, since $y(x)$ is a solution of (1), we have,

$$
d \phi(x) / d x=x^{-3 / 2} y(x)\left(\frac{1}{4}-x^{2} F\left(y^{2}(x), x\right)\right) .
$$

Now if we rewrite (1) as

$$
y^{\prime \prime}+\frac{1}{4 x^{2}} y=\frac{y}{4 x^{2}}\left(\frac{1}{4}-x^{2} F\left(y^{2}(x), x\right)\right),
$$

and apply the variation of constants formula, we obtain

(10) $y(x)=x^{1 / 2}\left(a+b \log \frac{x}{x_{1}}\right)+x^{1 / 2} \int_{x_{1}}^{x} s^{-3 / 2} \log \frac{x}{s}\left(\frac{1}{4}-s^{2} F\left(y^{2}(s), s\right) y(s)\right) d s$, where $a=x_{1}^{-1 / 2} y\left(x_{1}\right)$ and $b=\phi\left(x_{1}\right)$. Assume that $y$ is positive for $x \geqq x_{1}$ and that

$$
y(x)<(M x)^{1 / 2},
$$


when $x=x_{1}$. By continuity then (11) holds on some interval $\left[x_{1}, x_{2}\right)$, and on that interval we obtain, using (11) in (10),

$$
y(x) \leqq x^{1 / 2}\left(a+b \log \frac{x}{x_{1}}\right)+\frac{1}{4} M^{1 / 2} x^{1 / 2} \int_{x_{1}}^{x} s^{-1} \log \frac{x}{s} d s,
$$

or, using $a=x_{1}^{-1 / 2} y\left(x_{1}\right)<M^{1 / 2}$,

$$
y(x) \leqq x^{1 / 2}\left(M^{1 / 2}+b \log \frac{x}{x_{1}}+\frac{1}{8} M^{1 / 2}\left(\log \frac{x}{x_{1}}\right)^{2}\right),
$$

for $x_{1} \leqq x<x_{2}$. If the logarithmic polynomial

satisfies

$$
P(\xi)=M^{1 / 2}+b \log \xi+\frac{1}{8} M^{1 / 2}(\log \xi)^{2}
$$

$$
M^{1 / 2}>P(\xi)>0, \text { for } 1<\xi<A,
$$

then, by (12), the inequality (11) will hold also when $x=x_{2}$, provided $x_{2}<A x_{1}$. This last fact can be used to show that the validity of (12) extends to $\left[x_{1}, A x_{1}\right]$. Now let $A>1$ be given, there exists then a $B>0$, such that if $-b>B$ the logarithmic polynomial $P(\xi)$ defined above satisfies

$$
M^{1 / 2}>P(\xi)>0 \quad \text { for } 1 \leqq \xi<A^{\prime}, \quad P\left(A^{\prime}\right)=0,
$$

where $A^{\prime}=A^{\prime}(b)<A$; moreover $B$ depends only on $A$ and $M$. The foregoing remarks show that if $y$ is, as above, positive for $x \geqq x_{1}$ and satisfies (11) at $x=x_{1}$ then $\phi\left(x_{1}\right)>-B$. Since, in view of (4), the right-hand side of (9) is negative for $y(x) \geqq$ $(M x)^{1 / 2}$, it follows from (6) and (4) that $\phi(x)>-B$ for all $x \geqq x_{1}$. Repeating the same argument, but "going backwards" we see that if $x_{0}$ is the last zero of the eventually positive solution $y$, then for $x_{1}>A x_{0}$, and when (11) holds at $x=x_{1}$ we must have $\phi\left(x_{1}\right)<B$. Using (4), (6), and (9) as before we conclude that $\phi(x)<B$ for all large $x$. If $y(x)<0$ for all large $x$, then the above argument applied to the solution $-y(x)$ gives (8). This completes the proof.

LEMMA 3. If $y(x)$ is a solution of (1), then the function $\Psi(x)$ defined by

$$
\Psi(x)=x\left(y^{\prime 2}(x)+G\left(y^{2}(x), x\right)\right)-y(x) y^{\prime}(x)
$$

is nondecreasing in $x$.

Proof. We give a proof for the case where $F(t, x)$ is of class $C^{1}$. For the general case, the result will follow by a standard argument involving approximation of $F(t, x)$ by $C^{1}$ functions. By a straightforward computation we have the identity

$$
\frac{d}{d x} \Psi(x)=\left(2 x y^{\prime}(x)-y(x)\right)\left[y^{\prime \prime}(x)+y(x) F\left(y^{2}(x), x\right)\right]+Q\left(y^{2}, x\right),
$$

where $Q(t, x)=G(t, x)+t F(t, x)+x G_{x}(t, x)$. Note that the function $Q(t, x)$ satisfies

$$
Q(t, x)=\left.\frac{\partial}{\partial \sigma}\left(\sigma G\left(\frac{t \sigma}{x}, \sigma\right)\right)\right|_{\sigma=x},
$$


for arbitrary $t, x>0$. Thus, in view of the nondecreasing character of $x G(\alpha x, x)$ for $\alpha>0$, we have $Q(t, x) \geqq 0$, and the desired conclusion follows.

Proof of Theorem 1. Let $y(x)$ be a solution of (1), vanishing at $x_{1} \geqq x_{0}$, and let $x_{2}$ be some value of $x \geqq x_{1}$ for which

$$
x_{2}^{2} F\left(y^{2}\left(x_{2}\right), x_{2}\right) \leqq \frac{1}{4}+c .
$$

so that in particular from (4) we have

$$
y^{2}\left(x_{2}\right) \leqq M x_{2}
$$

Suppose that $y\left(x_{2}\right)>0$ and $y^{\prime}\left(x_{2}\right)>0$, then, by Lemma 3 , it follows

$$
x_{2}\left[y^{\prime 2}\left(x_{2}\right)+G\left(y^{2}\left(x_{2}\right), x_{2}\right)\right] \geqq C_{0},
$$

where the constant $C_{0}=x_{1} y^{2}\left(x_{1}\right)$. By (5), (13), and (14), we have

$$
x_{2} G\left(y^{2}\left(x_{2}\right), x_{2}\right) \leqq K x_{2} y^{2}\left(x_{2}\right) F\left(y^{2}\left(x_{2}\right), x_{2}\right) \leqq K M\left(\frac{1}{4}+c\right) .
$$

Suppose that $y(x)$ is any solution of (1) defined by the initial values $y\left(x_{1}\right)=0$ and $x_{1} y^{\prime 2}\left(x_{1}\right) \geqq B^{\prime 2}$, where $B^{\prime 2}>2 B^{2}+M / 2+\left(\frac{1}{4}+c\right) K M$, then, it follows from (15) and (16) that for arbitrary large $x_{2}$ for which (13) is satisfied,

$$
\begin{aligned}
\phi\left(x_{2}\right) & =x_{2}^{1 / 2} y^{\prime}\left(x_{2}\right)-\frac{1}{2} x_{2}^{-1 / 2} y\left(x_{2}\right) \\
& \geqq x_{2}^{1 / 2} y^{\prime}\left(x_{2}\right)-\frac{1}{2} M^{1 / 2} \\
& \geqq\left(B^{\prime 2}-K M\left(\frac{1}{4}+c\right)\right)^{1 / 2}-\frac{1}{2} M^{1 / 2}>B .
\end{aligned}
$$

If such a solution $y(x)$ is nonoscillatory then the above clearly contradicts (8); hence $y(x)$ is an oscillatory solution of (1).

3. Nonoscillation theorem. We shall now prove a result which is the desired counterpart of Theorem $\mathrm{C}$ for equation (2). The following result is also a generalization of Theorem $\mathrm{D}$.

THEOREM 2. Let. $F(t, x)$ and $G(t, x)$ be given as before. Suppose that there exists a constant $x_{0}>3$, such that for $x \geqq x_{0}$, we have (i) $F\left(t_{2}, x\right) \leqq F\left(t_{1}, x\right), t_{2} \leqq t_{1}$, and (ii) for every $\alpha>0$, the function $x \log x G(\alpha x \log x, x)$ is nonincreasing in $x$. Then equation (1) is nonoscillatory.

Before we prove Theorem 2, we need two preliminary lemmas which also seem to have independent interest in themselves. In the following, we restrict the independent variable $x$ to the half open interval $\left[x_{0}, \infty\right)$ without further mention.

LEMMA 4. Let $a_{1}$ be a zero of $y(x)$ satisfying (1), and $a_{2}>a_{1}$ such that $y\left(a_{2}\right) y^{\prime}\left(a_{2}\right)=0$. Then,

$$
a_{2} \log a_{2}\left(y^{\prime 2}\left(a_{2}\right)+G\left(y^{2}\left(a_{2}\right), a_{2}\right)\right)-a_{1} \log a_{1} y^{\prime 2}\left(a_{1}\right) \leqq-\int_{a_{1}}^{a_{2}} \frac{y(x) y^{\prime}(x)}{x} d x .
$$


Proof. Denote $Q(t, x)=G(t, x)+t F(t, x)+x G_{x}(t, x)$ as before and observe that

$$
(\log x) Q(t, x)+G(t, x)+t F(t, x)=\left.\frac{\partial}{\partial \sigma}\left(\sigma \log \sigma G\left(\frac{t \sigma \log \sigma}{x \log x}, \sigma\right)\right)\right|_{\sigma=x},
$$

for arbitrary $t, x>0$. Thus in view of the nonincreasing character of $(x \log x)$ - $G(\alpha x \log x, x)$ for $\alpha>0$, the left-hand side of (18) is nonpositive. Let $y(x)$ be a nontrivial solution of (1), and define $\Psi(x)$ by

$$
\Psi(x)=x\left(y^{\prime 2}(x)+G\left(y^{2}(x), x\right)\right)-y(x) y^{\prime}(x)
$$

as in Lemma 3. An easy computation shows

$$
\frac{d}{d x}(\log x \Psi(x))=\log x Q\left(y^{2}(x), x\right)+G\left(y^{2}(x), x\right)+y^{\prime 2}(x)-\frac{y(x) y^{\prime}(x)}{x} .
$$

Integrating (19) between $a_{1}$ and $a_{2}$ and using the relation

$$
\int_{a_{1}}^{a_{2}} y^{\prime 2}(x) d x=\int_{a_{1}}^{a_{2}} y^{2}(x) F\left(y^{2}(x), x\right) d x,
$$

we find

$$
\begin{aligned}
& \int_{a_{1}}^{a_{2}} \frac{d}{d x}(\log x \Psi(x)) d x \\
& \quad=\int_{a_{1}}^{a_{2}}\left[\log x Q\left(y^{2}, x\right)+G\left(y^{2}, x\right)+y^{2} F\left(y^{2}, x\right)\right] d x-\int_{a_{1}}^{a_{2}} \frac{y(x) y^{\prime}(x)}{x} d x,
\end{aligned}
$$

from which (17) readily follows.

LEMMA 5. Let a be any zero of $y(x)$ satisfying (1) and b be the first zero of $y^{\prime}(x)$ to the right of $a$. Then there exists a constant $B_{0}$, depending only on the first zero $a_{0}$ of $y(x)$ such that

$$
\int_{a}^{b} y^{\prime 2}(x) d x \leqq B_{0}
$$

Proof. With no loss of generality, we may assume $y(x)>0$ on $(a, b)$. For $x \in(a, b)$, we have

$$
\left((x-a) y^{\prime}-y\right)^{\prime}=(x-a) y^{\prime \prime}=-(x-a) y F\left(y^{2}, x\right)<0
$$

hence $y^{\prime}(x)(x-a) \leqq y(x)$. Using this estimate, we easily verify the following inequalities:

$$
\begin{aligned}
\int_{a}^{b} y^{\prime 2}(x) d x & =\int_{a}^{b} \frac{x-a+a}{x} y^{\prime 2}(x) d x \\
& \leqq \int_{a}^{b} \frac{y(x) y^{\prime}(x)}{x} d x+a \int_{a}^{b} \frac{y^{\prime 2}(x)}{x} d x
\end{aligned}
$$




$$
\begin{aligned}
\int_{a}^{b} \frac{y^{\prime 2}(x)}{x} d x & =\int_{a}^{b} \frac{x-a+a}{x^{2}} y^{\prime 2}(x) d x \\
& \leqq \int_{a}^{b} \frac{y(x) y^{\prime}(x)}{x^{2}} d x+a \int_{a}^{b} \frac{y^{\prime 2}(x)}{x^{2}} d x \\
& \leqq \frac{1}{a} \int_{a}^{b} \frac{y(x) y^{\prime}(x)}{x} d x+a \int_{a}^{b} \frac{y^{\prime 2}(x)}{x^{2}} d x
\end{aligned}
$$

Combining (21) and (22), and using the fact that $y^{\prime}(x)<y^{\prime}(a)$ for $x \in(a, b)$, we obtain

$$
\int_{a}^{b} y^{\prime 2}(x) d x \leqq 2 \int_{a}^{b} \frac{y(x) y^{\prime}(x)}{x} d x+a y^{\prime 2}(a) .
$$

Using (17) in (23), we have

$$
\begin{aligned}
\int_{a}^{b} y^{\prime 2}(x) d x & \leqq 2 a_{0} \log a_{0} y^{\prime 2}\left(a_{0}\right)+\frac{a_{0} \log a_{0} y^{\prime 2}\left(a_{0}\right)}{\log a} \\
& \leqq 3 a_{0} \log a_{0} y^{\prime 2}\left(a_{0}\right)=B_{0} .
\end{aligned}
$$

Proof of Theorem 2. Assume that the assertion is false, hence, there exists an oscillatory solution $y(x)$ of (1). Let $a_{0}, a_{1}, a_{2}, \ldots, a_{n}, \ldots$ be consecutive zeros of $y(x)$ and $b_{0}, b_{1}, b_{2}, \ldots, b_{n}, \ldots$ be zeros of $y^{\prime}(x)$ with $a_{k-1}<b_{k-1}<a_{k}, k=1,2,3, \ldots$ Define a sequence of positive numbers $\left\{\mu_{k}\right\}$ by

$$
\mu_{k}=\int_{a_{k}}^{b_{k}} y^{\prime 2}(x) d x=\int_{a_{k}}^{b_{k}} y^{2}(x) F\left(y^{2}(x), x\right) d x .
$$

For $x \in\left[a_{k}, b_{k}\right]$, we have by Schwarz's inequality

$$
y^{2}(x) \leqq\left(x-a_{k}\right) \int_{a_{k}}^{x} y^{\prime 2}(x) d x \leqq \mu_{k} x .
$$

Using (26) in (25) and the nondecreasing character of $F(t, x)$ with respect to $t$, we have

$$
1 \leqq \int_{a_{k}}^{b_{k}} x F\left(\mu_{k} x, x\right) d x \leqq \int_{a_{k}}^{b_{k}} x F\left(B_{0} x, x\right) d x,
$$

where $B_{0}$ is given by (20). Again by assumption (i), we know that $G(t, x)$ is convex in $t$. Hence,

$$
G(\beta x \log x, x)-G(\alpha x, x) \geqq(\beta \log x-\alpha) x F(\alpha x, x),
$$

provided $\beta \log x-\alpha \geqq 0$. Let $\beta=\alpha\left(\log x_{0}\right)^{-1}$, then (28) may be rewritten as

$$
G\left(\frac{\alpha x \log x}{\log x_{0}}, x\right) \geqq\left(\alpha \frac{\log x}{\log x_{0}}-\alpha\right) x F(\alpha x, x) .
$$

Choose $x_{1} \geqq x_{0}$ so that $\log x \geqq 2 \log x_{0}$ for $x \geqq x_{1}$. Now (29) becomes for $x \geqq x_{1}$

$$
G\left(\frac{\alpha x \log x}{\log x_{0}}, x\right) \geqq \frac{\alpha \log x}{2 \log x_{0}} x F(\alpha x, x) .
$$


Pick $k_{0}$ so that $a_{k} \geqq x_{1}$ for $k \geqq k_{0}$. Using (30) in (27), we observe

$$
\begin{aligned}
1 & \leqq \int_{a_{k}}^{b_{k}} x F\left(B_{0} x, x\right) d x \leqq \int_{a_{k}}^{b_{k}} \frac{2 \log x_{0}}{B_{0} \log x} G\left(B^{\prime} x \log x, x\right) d x \\
& =\frac{2}{B^{\prime}} \int_{a_{k}}^{b_{k}} \frac{1}{x(\log x)^{2}} x \log x G\left(B^{\prime} x \log x, x\right) d x,
\end{aligned}
$$

where $B^{\prime}=B\left(\log x_{0}\right)^{-1}$. Denote $G_{0}=a_{k_{0}} \log a_{k_{0}} G\left(B^{\prime} a_{k_{0}} \log a_{k_{0}}, a_{k_{0}}\right)$. Using assumption (ii), we obtain from (31) the following estimate valid for $k \geqq k_{0}$,

$$
1 \leqq \frac{2 G_{0}}{B_{1}} \int_{a_{k}}^{b_{k}} \frac{d x}{x(\log x)^{2}} \leqq \frac{2 G_{0}}{B^{\prime} \log a_{k}}
$$

Letting $k \rightarrow \infty$ in (32), we obtain the desired contradiction.

4. Discussion. Theorem 1 generalizes the result of Jasny and Kurzweil, i.e. Theorem A, in several directions. First, as remarked before, the function $F(t, x)$ is not necessarily of the form of a product of two functions each of $t$ and $x$ alone. Next, the usual assumption on $F(t, x)$ that it be nondecreasing in $t$ is weakened to condition (5) which admits a much larger class of functions. Finally, we would like to point out that in contrast to Theorem A which is strictly a nonlinear result, our Theorem 1 also covers the linear case as well. Consider equation (2) with $\gamma=1$. Assumptions (i) and (ii) thus reduce to: there exist constants $x_{0}, c>0$ such that

$$
x^{2} p(x) \geqq \frac{1}{4}+c, \quad x \geqq x_{0}
$$

and that $x^{2} p(x)$ is nondecreasing in $x$. We note that the second condition may be waived in general. Consider a function $\bar{p}(x)$ defined for each $x \geqq x_{0}$ by $x^{2} \bar{p}(x)$ $=\inf _{x \leqq s<\infty} s^{2} p(s) \geqq \frac{1}{4}+c$. Clearly $p(x) \geqq \bar{p}(x)$ and $x^{2} \bar{p}(x)$ is nondecreasing in $x$. Applying our result to the linear equation $y^{\prime \prime}+p(x) y=0$ and making use of the Sturm Comparison Theorem, we obtain that the linear equation $y^{\prime \prime}+p(x) y=0$ is oscillatory. In this way, Theorem 1 recovers the well-known result of Kneser [6] for the linear case, although it should be mentioned that in the proof presented here we have already used this linear result of Kneser, cf. Lemma 1. However, alternative proof may be devised so as to avoid the explicit use of Kneser's result. Such a proof necessarily involves longer arguments.

It is a simple matter to see that Theorem 2 includes the two results of Nehari [11] as special cases. In contrast to Theorem 1 whose proof is significantly different from that of Theorem A, here we make use of some of the techniques developed in [11] in establishing Theorems C and D. We remark that in both Theorems B and C, Kiguradze [5] and Nehari [11] stated their results only for equation (2) with $\gamma>1$, (in fact they assume $\gamma=2 n-1$, where $n$ is a positive integer $>1$ ). It is clear from Theorem 2 or a simple application of Sturm's Comparison Theorem that Theorems 
$\mathrm{B}$ and $\mathrm{C}$ remain valid for equation (2) in case $\gamma=1$. Indeed, the proofs of Kiguradze [7] and Nehari [11] made no explicit use of the fact that $\gamma>1$.

It should be mentioned that the proofs of both Theorems 1 and 2 can be made more transparent by transforming into an auxiliary equation. For example in Theorem 1 , let $t=\log x$ and $x^{-1 / 2} y(x)=u(t)$, then equation (1) is transformed into

$$
d^{2} u / d t^{2}+u\left(e^{2 t} F\left(e^{t} u^{2}, e^{t}\right)-\frac{1}{4}\right)=0 .
$$

Clearly equation (1) possesses an oscillatory solution if and only if equation (34) does. Since this approach does not shorten the presentation, we choose to use the present analysis in order to conform with Nehari's work. A similar comment applies to Theorem 2. Here we use the change of variables: $t=\log \log x$ and $(x \log x)^{-1 / 2} y(x)=u(t)$.

Previously it was tacitly assumed that every local solution of (1) can be extended to $[0, \infty)$. Actually this assumption can be omitted without essential modification of the statements or proofs of Theorems 1 and 2, provided that we understand by a solution of (1) a $C^{2}$ solution defined on a right maximal interval of existence $\left[a_{1}, \omega\right), a_{1} \geqq 0, \omega \leqq+\infty$. We shall call such a solution oscillatory if it has infinitely many zeros in $\left[x_{1}, \omega\right)$; if we adopt this definition then every solution $y$ having a finite right maximal interval of existence is oscillatory, see for example [2]. Moreover, if $a_{1}<a_{2}<\cdots<\omega<\infty$, are the zeros of the solution $y$ then $\lim _{n \rightarrow \infty}\left|y^{\prime}\left(a_{n}\right)\right|=\infty$, and also lim $\sup _{x \rightarrow \omega}|y(x)|=\infty$.

For Theorem 2 the assumption on the continuability of solutions is superfluous, since for a given solution $y(x), y^{\prime}(x)$ must remain bounded on its maximal interval of existence because of (17). In fact, Nehari [11] has already noted that every nonoscillatory solution is ipso facto continuable throughout $(a, \infty)$. We also note that the continuability assumption is not necessary in Theorem A, as it is already guaranteed by the hypothesis. In [4], Jasny assumes that $p$ is locally absolutely continuous and discusses the prolongability of solutions quite independent of the discussion here. (Cf. also Kurzweil [7], p. 358.) We note in fact that in these cases the monotonicity of $x^{\beta} p(x), \beta>0$, implies that $p(x)$ is locally of bounded variation, and hence the continuability of solutions follows from a result in [2].

We would like to point out that results in this paper may be stated in terms of the more general equation:

$$
y^{\prime \prime}+f(y, x)=0
$$

where $f(y, x)$ satisfies a set of conditions similar to those we impose on the function $F(t, x)$. Such a generalization does not seem to add much to the present knowledge of the problem, we content ourselves with just a mention of such a possibility.

Finally, we note that in contrast to results which guarantee oscillation of all solution or the existence of a nonoscillatory solution, our conditions on the growth of the function $G(t, x)$ are not in terms of convergence or divergence of a certain integral. In this sense, our results are not totally satisfactory. For example, Atkinson 
[1] has established a necessary and sufficient condition for equation (2), when $\gamma>1$, to be oscillatory, namely

$$
\int^{\infty} x p(x) d x=\infty
$$

(Extension of Atkinson's result to more general equations similar to (1) have been given by Macki and Wong [8].) It would be tempting to conjecture that a necessary and sufficient condition for equation (2) to be nonoscillatory is

$$
\int^{\infty} x^{(\gamma+1) / 2} p(x) d x<\infty .
$$

Thus far, the validity of such a conjecture remains unsettled.

\section{REFERENCES}

1. F. V. Atkinson, On second-order non-linear oscillations, Pacific J. Math. 5 (1955), 643-647. MR 17, 264.

2. C. V. Coffman and D. F. Ullrich, On the continuation of solutions of a certain non-linear differential equation, Monatsh. Math. 71 (1967), 385-392. MR 37 \#3078.

3. S. P. Hastings, Boundary value problems in one differential equation with a discontinuity, J. Differential Equations 1 (1965), 346-369. MR 31 \#4954.

4. M. Jasny, On the existence of an oscillating solution of the nonlinear differential equation of the second order $y^{\prime \prime}+f(x) y^{2 n-1}=0, f(x)>0$, Casopis Pěst. Mat. 85 (1960), 78-83. (Russian) MR 26 \#408.

5. I. T. Kiguradze, On the conditions for oscillation of solutions of the differential equation $u^{\prime \prime}+a(t)|u|^{n}$ sgn $u=0$, Casopis Pěst. Mat. 87 (1962), 492-495. (Russian) MR 31 \#6026.

6. A. Kneser, Untersuchungen über die reelen Nullstellen der Integrale linearer Differential Gleichungen, Math. Ann. 42 (1893), 409-435.

7. J. Kurzweil, A note on oscillatory solution of equation $y^{\prime \prime}+f(x) y^{2 n-1}=0$, Časopis Pěst. Mat. 85 (1960), 357-358. (Russian) MR 23 \#A3322.

8. J. W. Macki and J. S. W. Wong, Oscillation of solutions to second-order nonlinear differential equations, Pacific J. Math. 24 (1968), 111-117. MR 37 \#507.

9. R. A. Moore and Z. Nehari, Nonoscillation theorems for a class of nonlinear differential equations, Trans. Amer. Math. Soc. 93 (1959), 30-52. MR 22 \#2755.

10. Z. Nehari, On a class of nonlinear second-order differential equations, Trans. Amer. Math. Soc. 95 (1960), 101-123. MR 22 \#2756.

11. - A nonlinear oscillation problem, J. Differential Equations 5 (1969), 452-460.

12. J. S. W. Wong, On second order nonlinear oscillation, Funkcial. Ekvac. 11 (1969), 207-234.

Carnegie-Mellon University, Pittsburgh, Pennsylvania 